\title{
Experimental Validation of Dynamic Restoration in GMPLS-controlled Multi-layer Networks using PCE-based Global Concurrent Optimization
}

\author{
R. Martínez ${ }^{1}$, A. Castro ${ }^{2}$, R. Casellas ${ }^{1}$, R. Muñoz ${ }^{1}$, L. Velasco ${ }^{2}$, R. Vilalta ${ }^{1}, J_{\text {. Comellas }}{ }^{2}$ \\ ${ }^{1}$ Centre Tecnològic de Telecomunicacions de Catalunya (CTTC), Av. Carl Friedrich Gauss n7, 08860 Castelldefels, Spain \\ ${ }^{2}$ Universitat Politècnica de Catalunya (UPC), Jordi Girona 1-3, 08034 Barcelona, Spain \\ \{ricardo.martinez, ramon.casellas, raul.munoz, ricard.vilalta\}@cttc.es; \{acastro, lvelasco\}@ac.upc.edu; comellas@tsc.upc.edu
}

\begin{abstract}
An experimental validation of a PCE-based GCO architecture for dynamic restoration in GMPLS multi-layer networks is presented. This solution better addresses the concurrence of multiple connection requests favoring grooming objectives and attaining higher restorability. OCIS codes: (060.4261) Networks, protection and restoration; (060.4253) Networks, circuit-switched
\end{abstract}

\section{Introduction}

A multi-layer network (MLN) integrating both packet MPLS-TP and optical WSON switching is seen as a cost/energy efficient solution for deploying next-generation transport networks, leveraging the bandwidth flexibility and coarse transport capacity, provided by MPLS-TP and WSON, respectively. In this context, a GMPLS unified control plane [1], combined with a centralized PCE [2], is adopted to provide signaling, routing and path computation functions to automatically provision/restore Label Switched Paths (LSPs). This leads to attain an efficient use of the network resources exploiting MLN TE strategies where multiple packet LSPs are grouped/groomed over logical links (Forwarding Adjacencies, FA [1]) created by established optical LSPs.

MLN must be designed to be fault-tolerant to rapidly and efficiently recover disrupted LSPs [3]. We focus on dynamic restoration: failed LSPs are dynamically restored by a backup path computed by the PCE. Specifically, the ingress node (Path Computation Client, PCC) of each disrupted LSP sends a path request to the PCE. This may result on a bulk of path computations if multiple LSPs were interrupted. Each request is sequentially and independently served using as input the TE Database (TED) information (e.g., sniffing the OSPF-TE) and the particular path constraints (e.g., exclude failed links). Since the TED is not updated between path computations, the same resources may be assigned to different requests causing resource contention, and being unable to exploit the grooming objectives through new created logical links. Consequently, this PCE architecture attains a poor restorability. A solution to increase the restorability at the PCE relies on applying Global Concurrent Optimization (GCO) [4]. GCO aims at serving the bulk of path requests attaining the optimal solution for the whole network. Specifically, the path requests are served taking into account not only the collected TED information and particular path constraints, but also an auxiliary TED which is constructed to consider additional topology and resources (i.e., logical links) derived/resulting from the establishment of previously served paths within the same bulk. Although the latter information will not be reflected in the TED until those LSPs are successfully set up. Nevertheless, anticipating the potential network state may achieve a better path computation from a twofold perspective: on the one hand, the connection blocking caused by resource contention among multiple concurrent LSPs may be reduced; on the other hand, the grooming opportunities can be larger exploited since logical FA TE links induced by formerly computed paths are reused to route the subsequent paths within the same bulk. To do this, it is necessary to synchronize and delay the PCE responses to the PCCs to yield sufficient time to create the logical links associated to specific TE paths being usable by other subsequent paths. The main drawback of this approach, however, that it increases the response time affecting the overall restoration time.

Few works addressed the experimental application of the PCE-based GCO framework [5]. To the best of our knowledge, the present work is the first one on experimentally validating the dynamic restoration in a GMPLS MLN adopting a centralized PCE-based GCO. Besides presenting the problem description, the designed and implemented PCE-based GCO architecture is detailed, and finally assessed within the CTTC ADRENALINE testbed.

\section{Problem Description}

We assume that two Packet Switched Capable (PSC) LSPs (LSP1 and LSP2) are established between MPLS-TP nodes 1-5 and 6-5 through the WSON (Fig.1.a.). Two optical (Lambda SC, LSC) LSPs are thus set up through 1-23-4-5 and 6-7-8-3-4-5, respectively. Consequently, two logical packet FA TE links between the nodes 1-5 and 6-5 are induced [1,6]. Let us assume that an optical link failure occurs in link 3-4 (Fig.1.b) affecting both LSC LSPs. After the failure is optically detected, the ingress nodes (1 and 6) of the failed LSPs are notified to trigger the restoration mechanism. In such a MLN, a break-before-make strategy is applied tearing down the failed optical LSPs which then induce the elimination of their associated logical links at the packet layer [7]. Thus, the packet 
LSPs routed over such packet links fail. Next, the PCC embedded in each RSVP-TE controller of each failed PSC LSP sends a backup path computation request to the PCE specifying endpoints ( $s$ and $d$ ), required bandwidth profile (bw) and failed link (link 3-4). Applying a PCE with sequential computation (no GCO), it is likely that the computed backup paths may try to allocate the same resources (e.g., wavelengths) on specific links since path computations use the same TED which is not yet updated. Thereby, when signaling the LSPs (LSC LSP1 LSP2), this may cause resource contention (at link 7-8) attaining poor restorability (e.g., LSP1 is successfully restored but not LSP2).

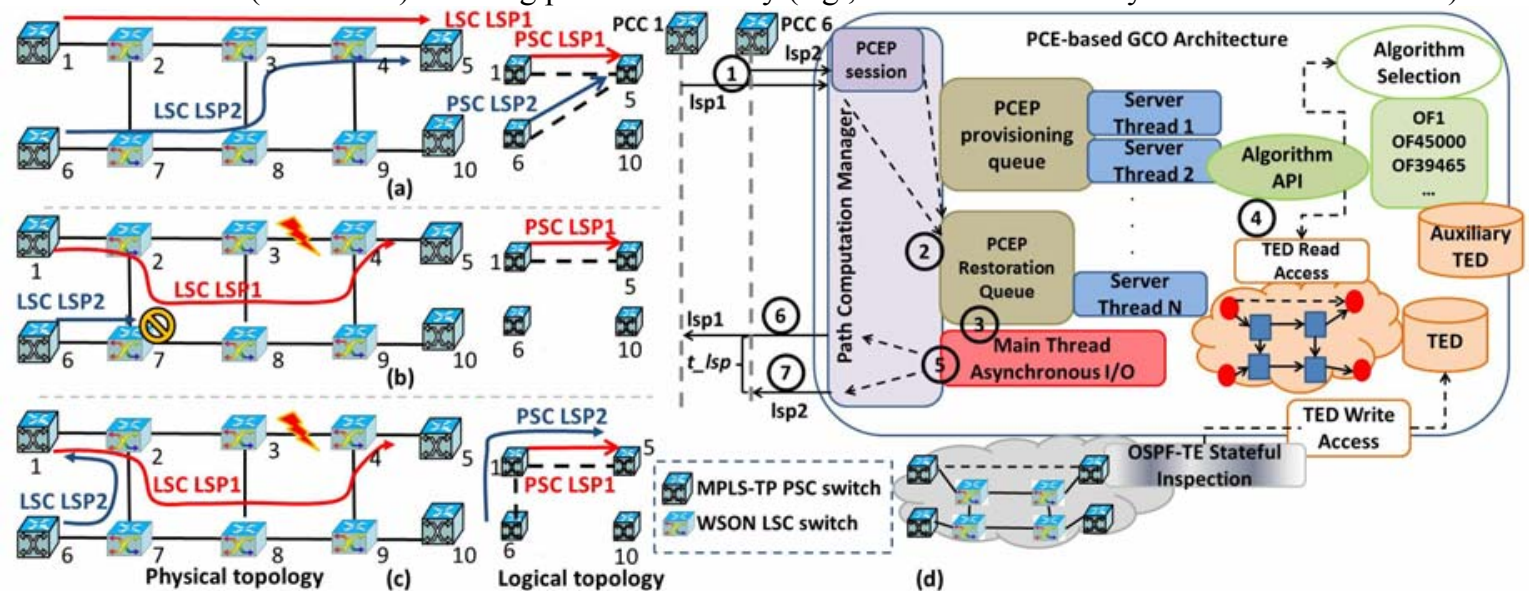

Fig.1. a.) Establishment of 2 packet LSPs in a MLN: physical and logical topologies. b) Failed restoration using a PCE applying sequential path computation. c) Successfully restoration using PCE-based GCO. d) Designed and implemented PCE-based GCO architecture.

To enhance the overall restorability of a received bulk of path computation requests, the PCE-based GCO is applied. Path computation requests are stored in a dedicated PCE buffer during a programmable timer, which is started upon reception of the first restoration request. When the timer expires, the requests are processed. To reduce the resource contention and favor grooming objectives, the GCO enables that, when serving a request, the algorithm considers also the upcoming network status changes besides the TED (e.g., occupied wavelengths) along with the induction of potential new logical links considering the computed paths within the targeted bulk. In Fig 1.c, the backup path for LSP1 is 1-2-7-8-9-4-5, which will induce a logical link between nodes 1-5. The wavelengths and logical link to be used/created when LSP1 is set up are pre-considered for computing the LSP2 path, even if LSP1 has not been yet signaled. Consequently, LSP2 can leverage (groom into) the logical link between nodes 1-5 that will be induced after establishing LSP1. In the example, the LSP2 path is thus formed by: an optical segment through 6-7-2-1 (which will induce its own logical link between nodes 6-1) and grooming over the mentioned logical link connecting nodes 1-5. As a result both LSP1 and LSP2 can be successfully restored. The main restriction is that LSP2 is dependent on the establishment of LSP1 restoration path. Therefore, the signaling of LSP2 is delayed to guarantee that LSP1 is established. This is done delaying the PCE response to LSP2 ingress PCC.

\section{PCE Architecture in Support of Global Concurrent Optimization}

Fig.1.d depicts the architecture of the implemented PCE-based GCO. For the LSP provisioning requests or disrupted LSPs, the ingress PCC sends a path request via the PCE protocol (PCEP) (see step1). At the PCE, the request is stored in either the provisioning or restoration queues. If the path request contains the eXclude Route Object (XRO), conveying the failed link with the fail-bit active, it is stored in the restoration queue. For regular provisioning, the request is stored in a priority queue served by a thread pool. The selected routing algorithm is determined by the objective function (OF) object included in the PCEP request. In this work, the MLN routing algorithm computes a modified shortest path which minimizes the resources use (i.e., wavelengths) leveraging the grooming objectives.

Restoration requests are grouped (step2) and processed after a configurable timer (t_queue, step3) expires. This allows grouping and synchronizing the backup paths forming the bulk. Once $t$ queue expires, it triggers the selected restoration algorithm (step4). To this end, the algorithm uses an auxiliary TED where the input parameters are the pair $s-d$, bw, and the failed link/s (XRO) to be excluded. The auxiliary TED is initially constructed duplicating the current regular TED. Nevertheless, as paths requests are computed, the resources along with the potential logical links to be used by those TE paths are updated in the auxiliary TED for the subsequent path request to be processed. In addition, the algorithm specifies the ordering, sequence and timing of the PCEP responses, (step5) i.e., the PCE decides whether a PCEP response is immediately sent back to the PCC or delayed. The latter occurs when the computed path (e.g., LSP2 in the above example) presents some dependencies (e.g., the creation of a new logical link) with respect to a formerly computed path (e.g., LSP1). In this situation, the PCEP response is delayed ( $\left.t \_l s p\right)$ to guarantee that, for instance, the required logical link/s is actually induced/created (steps6, 7). 


\section{Experimental Validation}

The GMPLS/PCE control plane platform of the ADRENALINE testbed is used for experimentally validating the implemented PCE-based dynamic restoration with GCO for MLN. Fig.2.a. shows the topology formed by 10 PSC and 12 LSC nodes. Each PSC node is physically connected to its associated LSC node with 16 bidirectional ports. The optical links support $16 \mathrm{WDM}$ channels per direction operating at $10 \mathrm{Gbps}$. We consider a dynamic stochastic model for both PSC LSPs and link failure events. The arrival of the PSC requests and failure events are Poisson processes while the holding times of the LSPs / duration of the failures follow negative exponential distributions. The offered traffic load is set to 50 and 100 Er., with an average inter-arrival time set to $10 \mathrm{~s}$. Traffic is uniformly distributed among the PSC nodes requesting LSPs of $1 \mathrm{Gbps}$. The average failure inter-arrival time is set to 40s and the mean repair time set to 50s. Link failures are uniformly distributed exclusively among the optical (LSC) TE links. Each performance value is obtained requesting 2500 PSC LSPs. The PCE is co-located at node 13 (Vienna).

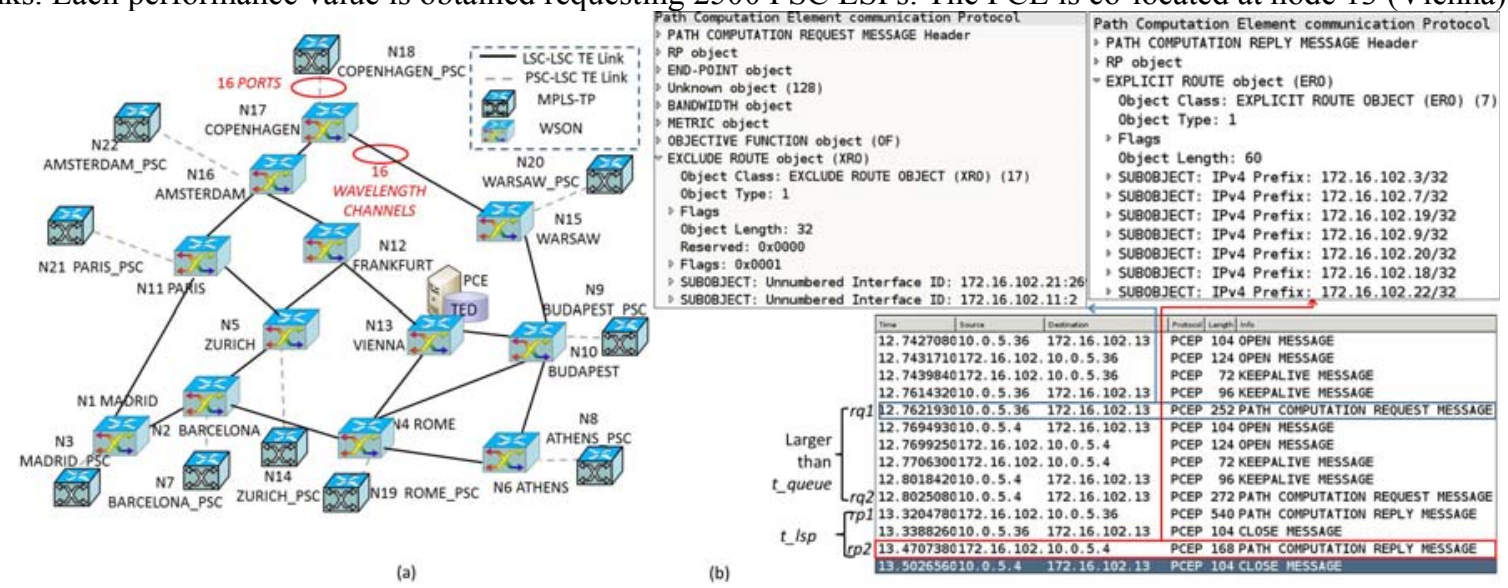

Fig.2. a. European physical MLN network topology. b. Packet messages exchange between PCC and PCE applying PCE-based GCO.

Exchanged PCEP request / reply messages for MLN restoration are shown in Fig.2.b. After several tests, $t$ _queue and $t$ lsp (difference between $r p 1$ and $r p 2$ ) were set to $100 \mathrm{~ms}$ and $150 \mathrm{~ms}$ (respectively) to allow delaying the PCEP replies without excessively penalizing the restoration time. Recall that $t$ queue is reset upon receiving rq1.

Tab.1 gathers the performance metric results using either sequential or GCO (bulk) path computation when restoring paths. We observe that for both traffic loads (50 and $100 \mathrm{Er}$ ), applying GCO does improve both the restorability and the BP with respect to the sequential strategy. Indeed, using GCO, the computed resources (e.g., new induced logical links) are more efficiently reused among the paths of a bulk. This allows both saving network resources (e.g., wavelength channels) and reducing the resource contention when signaling the LSPs. Consequently, the general LSP provisioning is also enhanced lowering the BP. Such benefits, however, are attained at the expenses of increasing the backup path computation latency, and thus restoration time, due to the use of $t$-queue and $t$ llsp for creating/processing the bulk and delaying the dependent paths, respectively. Therefore, a trade-off between performance (restorability and BP) and restoration time is stated when applying the PCE-based GCO restoration.

Tab 1. Performance metrics

\begin{tabular}{|c|c|c|c|c|c|c|c|}
\hline & Er. & $\begin{array}{l}\text { Working } \\
\text { Comp. (ms) }\end{array}$ & $\begin{array}{l}\text { Working Setup Delay } \\
(\mathrm{ms})\end{array}$ & $\begin{array}{l}\mathrm{BP} \\
(\%)\end{array}$ & Rest. Path Comp. (ms) & $\begin{array}{ll}\begin{array}{l}\text { Rest. } \\
(\mathrm{ms})\end{array} & \text { Time } \\
\end{array}$ & $\begin{array}{l}\text { Restorability } \\
(\%)\end{array}$ \\
\hline \multirow[t]{2}{*}{ Sequential } & 50 & 156 & 193 & 6.9 & 495 & 514 & 70.5 \\
\hline & 100 & 155.4 & 188.2 & 8.7 & 543 & 584 & 67.4 \\
\hline \multirow{2}{*}{$\begin{array}{ll}\text { Bulk } & - \\
\text { GCO }\end{array}$} & 50 & 150 & 192.5 & 0.6 & 912 & 940 & 91.5 \\
\hline & 100 & 158 & 191.1 & 6 & 1031 & 1100 & 82 \\
\hline
\end{tabular}

\section{Conclusions}

This work presents the implemented PCE GCO architecture for dynamic restoration in GMPLS MLN. The goal is to improve the general restorability of the MLN. We have experimentally validated the solution in the ADRENALINE testbed, and compared to a PCE strategy which attends sequentially backup paths (no global optimization). The PCE-based GCO strategy leads to attain an efficient reuse of the network resources among the paths of a bulk, which does increase considerably the restorability at the expenses of delaying the restoration time.

\section{Acknowledgments} Spanish MINECO projects DORADO (TEC2009-07995), FARO (TEC2012-38119), ELASTIC (TEC2011-27310).

\section{References}

[1] K. Shiomoto, et. al., IETF RFC 5212, 2008.

[2] A. Farrel, et. al., IETF RFC 4655, 2006

[3] P. Chołda and A. Jajszczyk, IEEE/OSA JLT, 28, 2010.
[4] Y. Lee, et. al., IETF RFC 5557, 2009.

[5] J. Ahmed, et. al., IEEE/OSA JLT, 30, 2012. 